CUBO A Mathematical Journal Vol.16, $N^{\underline{O}} 01$, (01-07). March 2014

\title{
Stationary Boltzmann equation and the nonlinear alternative of Leray-Schauder type
}

\author{
Rafael Galeano Andrades, Pedro Ortega Palencia \\ and \\ John Fredys Cantillo Palacio \\ Institute of Applied Mathematics, \\ Universidad de Cartagena, \\ Cartagena, Colombia. \\ rgaleanoa@unicartagena.edu.co, portegap@unicartagena.edu.co, \\ jcantillop@unicartagena.edu.co
}

\begin{abstract}
By applying a nonlinear alternative of Leray-Schauder type, a fixed point of an operator is found, which, in turn, comes to be a solution of stationary Boltzmann equation with boundary conditions of Maxwellian type.
\end{abstract}

\section{RESUMEN}

Aplicando una alternativa no lineal del tipo Leray-Schauder, se encontró un punto fijo de un operador, el cual corresponde a la solución de una ecuación de Boltzmann estacionaria con condiciones de frontera del tipo Maxwelliano.

Keywords and Phrases: Nonlinear alternative of Leray-Schauder type, Fixed point, Solution of stationary Boltzmann equation.

2010 AMS Mathematics Subject Classification: 35Q20. 


\section{Introduction}

Let us consider the Banach space $E=\left\{u \in L^{1}\left(\Omega \times B_{3 R}(0)\right): v_{i} \frac{\partial u}{\partial x_{i}} \in L^{1}\left(\Omega \times B_{3 R}(0)\right)\right\}$ with norm $\|u\|_{E}:=\|u\|_{L^{1}\left(\Omega \times B_{3 R}(0)\right)}+\left\|v_{i} \frac{\partial u}{\partial x_{i}}\right\|_{L^{1}\left(\Omega \times B_{3 R}(0)\right)}$ and we expect to find $u(x, v) \geq 0$, such that $\left\{\begin{array}{l}v \cdot \nabla_{\chi} u=Q(u, u), \quad u \in B_{E}(0, R) \\ u(x, v)=M(v)=e^{-|v|^{2}} \text { (Maxwellian), } u \in \partial B_{E}(0, R),(R>0)\end{array}\right.$

Here $\quad \mathrm{Q}(u, u)(v)=\int_{\mathrm{B}_{3 \mathrm{R}}(0)} \int_{|\mathrm{p}|=1}[p \cdot(v-z)] p\left[u\left(x, z^{\prime}\right) \mathfrak{u}\left(x, v^{\prime}\right)-\mathfrak{u}(x, z) u(x, v)\right] d p d z$,

is the collision operator, $\Omega$ bounded and regular and the speeds related by the following relations:

$$
\left\{\begin{array}{l}
v^{\prime}=v-[p \cdot(v-z)] p \\
z^{\prime}=z+[p \cdot(v-z)] p
\end{array}\right.
$$

$(z, v)$ and $\left(z^{\prime}, v^{\prime}\right)$ son the pre-collision and post-collision speeds, respectively. It can be noted that if $z, v \in \mathrm{B}_{\mathrm{R}}(0)$ in $\mathbb{R}^{n}$, then $z^{\prime}, v^{\prime} \in \mathrm{B}_{3 \mathrm{R}}(0)$.

Here, the following problem will be proved.

Theorem 1.1. Let us suppose that $\mathrm{Q}(\mathrm{u}, \mathrm{u}) \in \mathrm{B}_{\mathrm{E}}(\mathrm{u}, \mathrm{R} / 2) ; v_{i} \frac{\partial u}{\partial x_{i}} \in \mathrm{B}_{\mathrm{E}}(0, \mathrm{R} / 2 n) \quad$ y $\quad v_{i} \frac{\partial u_{n}}{\partial x_{i}} \in$ $\mathrm{B}_{\mathrm{E}}\left(v_{i} \frac{\partial u}{\partial x_{i}}, \mathrm{R}^{* *}\right), \mathrm{R}^{* *} \geq 0$, for $\mathrm{n}=1,2, \ldots$, moreover $0<\int_{\mathrm{B}_{3 \mathrm{R}}(0)} \int_{|\mathrm{p}|=1}\|v-z\| \mathrm{dpd} z<\infty$ and $0<\int_{\mathrm{B}_{3 \mathrm{R}}(0)} \int_{|\mathrm{p}|=1}\|v-z\| \mathrm{dpd} v<\infty$, then there exits a solution for $\mathrm{u} \in \mathrm{B}_{\mathrm{E}}(0, \mathrm{R})$ of problem (1.1).

In these stationary problems, the flows of quantities as entropy control and compactness properties are under control, but they do not imply, per se, the desired results. Anyway, energy control and similar properties are available from momentum flows and mass control that can be imposed on the problem to replace entropy-bounding non-availability. There are controls based on involution of entropy dissipation..

Using such tools, in the last years Arkeryd L. and Noury A., [2] - 3] - [4]- [5, have made a development focused on the results of solutions existence in the $\mathrm{L}^{1}$ context for nonlinear equations Boltzmann type and also for those presenting maxwellian equilibrium. The case of perturbation on the global maxwellian equilibrium has been typically studied since the 60's. Methods of general type as Hilbert spaces and contraction mapping have been used, being the pioneers [6] - [7] - [9] -[10]; in 11] exposed, generally discussed the problem of boundary value for the stationary equation, in [12] and [14] is proved the theorem for the stationary equation Povzner with certain spatial boundary conditions of type Maxwellian and in [13] there are applications to dynamics of fluids, we present the main result five lemmas. 


\section{Development}

The problem (1.1), $v \cdot \nabla_{x} \mathfrak{u}=\mathrm{Q}(\mathfrak{u}, \mathfrak{u})$ is equivalent to $\mathfrak{u}+v \cdot \nabla_{x} \mathfrak{u}=\mathfrak{u}+\mathrm{Q}(\mathfrak{u}, \mathfrak{u})$, this implies that $\mathfrak{u}=\mathfrak{u}+v \cdot \nabla_{x} \mathfrak{u}-\mathrm{Q}(\mathfrak{u}, \mathfrak{u})$ which suggests the following operator:

$$
\mathrm{J}(\mathrm{u}):=u+v \cdot \nabla_{x} u-Q(u, u)
$$

Defined on

$$
E=\left\{u \in L^{1}\left(\Omega \times B_{3 R}(0)\right): v_{i} \frac{\partial u}{\partial x_{i}} \in L^{1}\left(\Omega \times B_{3 R}(0)\right)\right\},
$$

$E$ is a Banach space with the norm

$$
\|u\|_{E}:=\|u\|_{L^{1}\left(\Omega \times B_{3 R}(0)\right)}+\left\|v_{i} \frac{\partial u}{\partial x_{i}}\right\|_{L^{1}\left(\Omega \times B_{3 R}(0)\right)}
$$

Finding fixed points of J, coincides with finding solutions of (1.1). So we will work to find fixed points, via alternative Leray-Schauder type, in effect:

Let $C=\overline{B_{E}(0, R)}$, this is a convex and closed set in $E$ and $U:=B_{E}(0, R)$, open ball centered in 0 and radius $R$.

Lemma 2.1. Let us suppose that $\mathrm{Q}(\mathrm{u}, \mathrm{u}) \in \mathrm{B}_{\mathrm{E}}(\mathrm{u}, \mathrm{R} / 2)$ and $v_{\mathrm{i}} \frac{\partial \mathrm{u}}{\partial \mathrm{x}_{\mathrm{i}}} \in \mathrm{B}_{\mathrm{E}}(0, \mathrm{R} / 2 \mathrm{n}), \mathfrak{u}=1,2, \ldots$, then $\mathrm{J}$ sends $\mathrm{C}$ in $\mathrm{C}$.

Proof. Let $u \in C=\overline{\mathrm{B}_{\mathrm{E}}(0, \mathrm{R})}$, as $\mathrm{J}(\mathfrak{u}):=\mathfrak{u}+v \cdot \nabla_{\mathrm{x}} \mathrm{u}-\mathrm{Q}(\mathrm{u}, \mathfrak{u})$, then

$$
\begin{aligned}
& |J(\mathfrak{u})| \leq|\mathfrak{u}-\mathrm{Q}(\mathfrak{u}, \mathfrak{u})|+\left|v \cdot \nabla_{x} \mathfrak{u}\right|=|\mathfrak{u}-\mathrm{Q}(\mathfrak{u}, \mathfrak{u})|+\sum_{i=1}^{\mathfrak{n}}\left|v_{i} \frac{\partial \mathfrak{u}}{\partial x_{\mathfrak{i}}}\right|, \quad \text { i.e, } \\
& \|J(u)\|_{L^{1}\left(\Omega \times B_{3 R}(0)\right)} \leq\|u-Q(u, u)\|_{L^{1}\left(\Omega \times B_{3 R}(0)\right)}+\sum_{i=1}^{n}\left\|v_{i} \frac{\partial u}{\partial x_{i}}\right\|_{L^{1}\left(\Omega \times B_{3 R}(0)\right)}
\end{aligned}
$$

now:

$$
\begin{gathered}
\frac{\partial J(u)}{\partial x_{i}}=\frac{\partial u}{\partial x_{i}}+\frac{\partial}{\partial x_{i}}\left[\sum_{i=1}^{n} v_{i} \frac{\partial u}{\partial x_{i}}\right]+\frac{\partial Q(u, u)}{\partial x_{i}}, \text { then } \\
\qquad \frac{\partial J(u)}{\partial x_{i}}|\leq| \frac{\partial}{\partial x_{i}}(u-Q(u, u))|+| \frac{\partial}{\partial x_{i}} \sum_{i=1}^{n} v_{i} \frac{\partial u}{\partial x_{i}} \mid, \text { i.e., } \\
\qquad\left\|\frac{\partial J(u)}{\partial x_{i}}\right\|_{L^{1}\left(\Omega \times B_{B R}(0)\right)} \leq\left\|\frac{\partial}{\partial x_{i}}(u-Q(u, u))\right\|_{L^{1}\left(\Omega \times B_{B R}(0)\right)}+\sum_{i=1}^{n}\left\|\frac{\partial}{\partial x_{i}}\left(v_{i} \frac{\partial u}{\partial x_{i}}\right)\right\|_{L^{1}\left(\Omega \times B_{3 R}(0)\right)} .
\end{gathered}
$$

from (2.1) and (2.2) we conclude that:

$$
\|J(u)\|_{E} \leq\|\mathfrak{u}-\mathrm{Q}(\mathfrak{u}, \mathfrak{u})\|_{E}+\sum_{i=1}^{n}\left\|v_{i} \frac{\partial u}{\partial x_{i}}\right\|_{E} \leq \frac{R}{2}+\frac{R}{2}=R,
$$

that is to say, $J(u) \in C=B_{E}(0, R)$. 
Lemma 2.2. If $\mathrm{u}_{\mathrm{n}} \in \mathrm{B}_{\mathrm{E}}(\mathrm{u}, \mathrm{R})$, such that $\left|\mathrm{u}_{\mathrm{n}}(\mathrm{x}, v)\right| \leq \mathrm{R},|\mathrm{u}(\mathrm{x}, \boldsymbol{v})| \leq \mathrm{R}$, for every $\mathrm{n}, \mathrm{x} \in \Omega$, $v \in \mathrm{B}_{3 \mathrm{R}}(0)$, moreover

$$
0<\int_{\mathrm{B}_{3 \mathrm{R}}} \int_{|\mathrm{p}|=1}\|(v-z)\| \mathrm{dpd} z<\infty \quad y \quad 0<\int_{\mathrm{B}_{3 \mathrm{R}}} \int_{|\mathrm{p}|=1}\|(v-z)\| \mathrm{d} \mathrm{d} \mathrm{d} v<\infty,
$$

then $\mathrm{Q}\left(\mathrm{u}_{\mathrm{n}}, \mathrm{u}_{\mathrm{n}}\right) \in \mathrm{B}_{\mathrm{E}}\left(\mathrm{Q}(\mathrm{u}, \mathrm{u}), \mathrm{r}^{*} \mathrm{R}\right)$ with

$$
\mathrm{r}^{*}=4 \mathrm{R} \max \left\{\int_{\mathrm{B}_{3 \mathrm{R}}} \int_{|\mathrm{p}|=1}\|(v-z)\| \mathrm{dpd} z, \int_{\mathrm{B}_{3 \mathrm{R}}} \int_{|\mathrm{p}|=1}\|(v-z)\| \mathrm{d} p \mathrm{~d} v\right\} . \quad\left(\mathrm{r}^{*} \geq 0\right)
$$

Proof. $\quad$ By definition of $\mathrm{Q}(\mathrm{u}, \mathrm{u})$, leads to

$$
\begin{aligned}
\left|\mathrm{Q}\left(\mathrm{u}_{n}, u_{n}\right)(v)-Q(u, u)(v)\right| & =\mid \int_{B_{3 R}(0)} \int_{|p|=1}[p \cdot(v-z)] p\left[u_{n}\left(x, z^{\prime}\right) u_{n}\left(x, v^{\prime}\right)-u_{n}(x, z) u_{n}(x, v)\right] d p d z \\
& -\int_{B_{3 R}(0)} \int_{|p|=1}[p \cdot(v-z)] p\left[u\left(x, z^{\prime}\right) \mathfrak{u}\left(x, v^{\prime}\right)-u(x, z) u(x, v)\right] d p d z \mid,
\end{aligned}
$$

That is to say:

$$
\begin{aligned}
& \left|\mathrm{Q}\left(\mathrm{u}_{\mathrm{n}}, \mathrm{u}_{\mathrm{n}}\right)(v)-\mathrm{Q}(\mathrm{u}, \mathrm{u})(v)\right| \\
& =\mid \int_{B_{3 R}(0)} \int_{|p|=1}[p \cdot(v-z)] p\left[u_{n}\left(x, z^{\prime}\right) u_{n}\left(x, v^{\prime}\right)-u\left(x, z^{\prime}\right) u\left(x, v^{\prime}\right)\right. \\
& \left.-u_{n}(x, z) u_{n}(x, v)+u(x, z) u(x, v)\right] d p d z \\
& \leq \int_{\mathrm{B}_{3 \mathrm{R}}(0)} \int_{|\mathrm{p}|=1}|\mathrm{p} \cdot(v-z)| \mid \mathrm{u}_{\mathrm{n}}\left(x, z^{\prime}\right) \mathrm{u}_{\mathrm{n}}\left(x, v^{\prime}\right)-\mathrm{u}_{\mathrm{n}}\left(x, z^{\prime}\right) \mathfrak{u}\left(x, v^{\prime}\right) \\
& +u_{n}\left(x, z^{\prime}\right) \mathfrak{u}\left(x, v^{\prime}\right)-\mathfrak{u}\left(x, z^{\prime}\right) \mathfrak{u}\left(x, v^{\prime}\right)+\mathfrak{u}(x, z) \mathfrak{u}(x, v) \\
& -\mathfrak{u}(x, z) u_{n}(x, v)+u(x, z) u_{n}(x, v)-u_{n}(x, z) u_{n}(x, v) \mid d p d z
\end{aligned}
$$

then

$$
\begin{aligned}
& \left|\mathrm{Q}\left(\mathrm{u}_{n}, u_{n}\right)(v)-\mathrm{Q}(u, u)(v)\right| \\
& \leq \int_{\mathrm{B}_{3 \mathrm{R}}(0)} \int_{|\mathfrak{p}|=1}|p \cdot(v-z)|\left[\left|u_{n}\left(x, z^{\prime}\right)\right|\left|u_{n}\left(x, v^{\prime}\right)-\mathfrak{u}\left(x, v^{\prime}\right)\right|\right. \\
& \quad+\left|u\left(x, v^{\prime}\right)\right|\left|u_{n}\left(x, z^{\prime}\right)-\mathfrak{u}\left(x, z^{\prime}\right)\right|+|u(x, z)|\left|u_{n}(x, v)-u(x, v)\right| \\
& \left.\quad+\left|u_{n}(x, v)\right|\left|u_{n}(x, z)-u(x, z)\right|\right] d p d z
\end{aligned}
$$


so,

$$
\begin{aligned}
& \left\|\mathrm{Q}\left(u_{n}, u_{n}\right)-Q(u, u)\right\|_{L^{1}\left(\Omega \times B_{3 R}(0)\right)} \leq \int_{\Omega_{\Omega}} \int_{B_{3 R}(0)}\left|Q\left(u_{n}, u_{n}\right)(v)-Q(u, u)(v)\right| d x d v \\
& \leq \int_{\bar{\Omega}} \int_{\mathrm{B}_{3 \mathrm{R}}(0)} \int_{\mathrm{B}_{3 \mathrm{R}}(0)} \int_{|\mathrm{p}|=1}|p \cdot(v-z)|\left|\mathrm{u}_{n}\left(x, z^{\prime}\right)\right|\left|u_{n}\left(x, v^{\prime}\right)-u\left(x, v^{\prime}\right)\right| \mathrm{d} p \mathrm{~d} z \mathrm{~d} v \mathrm{~d} x \\
& +\int_{\bar{\Omega}} \int_{\mathrm{B}_{3 \mathrm{R}}(0)} \int_{\mathrm{B}_{3 \mathrm{R}}(0)} \int_{|\mathrm{p}|=1}\|v-z\|\left|\mathfrak{u}\left(x, v^{\prime}\right)\right|\left|\mathfrak{u}_{\mathrm{n}}\left(x, z^{\prime}\right)-\mathfrak{u}\left(x, z^{\prime}\right)\right| \mathrm{d} p \mathrm{~d} z \mathrm{~d} v \mathrm{~d} x \\
& +\int_{\bar{\Omega}} \int_{\mathrm{B}_{3 \mathrm{R}}(0)} \int_{\mathrm{B}_{3 \mathrm{R}}(0)} \int_{|\mathrm{p}|=1}\|v-z\||\mathrm{u}(x, z)|\left|\mathrm{u}_{\mathrm{n}}(x, v)-\mathfrak{u}(x, v)\right| \mathrm{d} p \mathrm{~d} z \mathrm{~d} v \mathrm{~d} x \\
& +\int_{\bar{\Omega}} \int_{\mathrm{B}_{3 \mathrm{R}}(0)} \int_{\mathrm{B}_{3 \mathrm{R}}(0)} \int_{|\mathrm{p}|=1}\|v-z\|\left|\mathrm{u}_{\mathrm{n}}(x, z)-\mathfrak{u}(x, z)\right| \mathrm{d} p \mathrm{~d} z \mathrm{~d} v \mathrm{~d} x \\
& \leq \int_{\mathrm{B}_{3 \mathrm{R}}(0)} \int_{|\mathrm{p}|=1} \mathrm{R}\|v-z\| \mathrm{d} p \mathrm{~d} z \int_{\bar{\Omega}} \int_{\mathrm{B}_{3 \mathrm{R}}(0)}\left|\mathrm{u}_{\mathrm{n}}\left(x, v^{\prime}\right)-u\left(x, v^{\prime}\right)\right| \mathrm{d} v \mathrm{~d} x \\
& +\int_{\mathrm{B}_{3 \mathrm{R}}(0)} \int_{|\mathrm{p}|=1} \mathrm{R}\|v-z\| \mathrm{d} p \mathrm{~d} v \int_{\bar{\Omega}} \int_{\mathrm{B}_{3 \mathrm{R}}(0)}\left|\mathrm{u}_{\mathrm{n}}\left(x, z^{\prime}\right)-\mathrm{u}\left(x, z^{\prime}\right)\right| \mathrm{d} z \mathrm{~d} x \\
& +\int_{\mathrm{B}_{3 \mathrm{R}}(0)} \int_{|\mathfrak{p}|=1} \mathrm{R}\|v-z\| \mathrm{d} p \mathrm{~d} z \int_{\bar{\Omega}} \int_{\mathrm{B}_{3 \mathrm{R}}(0)}\left|\mathrm{u}_{\mathrm{n}}(x, v)-\mathfrak{u}(x, v)\right| \mathrm{d} v \mathrm{~d} x \\
& +\int_{\mathrm{B}_{3 \mathrm{R}}(0)} \int_{|p|=1} \mathrm{R}\|v-z\| \mathrm{dpd} v \int_{\Omega_{\Omega}} \int_{\mathrm{B}_{3 \mathrm{R}}(0)}\left|\mathrm{u}_{\mathrm{n}}(x, z)-\mathrm{u}(x, z)\right| \mathrm{d} z \mathrm{~d} x
\end{aligned}
$$

Making the change of variables $v^{\prime} \rightarrow v$ y $z^{\prime} \rightarrow z$, whose Jacobians are 1 , then:

$$
\begin{aligned}
& \left\|Q\left(u_{n}, u_{n}\right)-Q(u, u)\right\|_{L^{1}\left(\Omega \times B_{3 R}(0)\right)} \\
& \leq 2 \mathrm{R}\left[\int_{\mathrm{B}_{3 \mathrm{R}}(0)} \int_{|\mathrm{p}|=1}\|v-z\| \mathrm{dpd} z+\int_{\mathrm{B}_{3 \mathrm{R}}(0)} \int_{|p|=1}\|v-z\| \mathrm{dpd} v\right]\left\|u_{n}-u\right\|_{\mathrm{L}^{1}\left(\Omega \times \mathrm{B}_{3 \mathrm{R}}(0)\right)} \\
& \leq 2 \mathrm{R}\left[\int_{\mathrm{B}_{3 \mathrm{R}}(0)} \int_{|p|=1}\|v-z\| \mathrm{d} p \mathrm{~d} z+\int_{\mathrm{B}_{3 \mathrm{R}}(0)} \int_{|\mathrm{p}|=1}\|v-z\| \mathrm{d} p \mathrm{~d} v\right]\left\|\mathrm{u}_{\mathrm{n}}-\mathrm{u}\right\|_{\mathrm{E}} \\
& \leq 4 R \max \left\{\int_{B_{3 R}} \int_{|p|=1}\|(v-z)\| \mathrm{dpd} z, \int_{B_{3 R}} \int_{|p|=1}\|(v-z)\| \mathrm{d} p \mathrm{~d} v\right\}\left\|\mathrm{u}_{n}-u\right\|_{\mathrm{E}} .
\end{aligned}
$$

Hence $\mathrm{Q}\left(\mathrm{u}_{\mathrm{n}}, \mathrm{u}_{\mathrm{n}}\right) \in \mathrm{B}_{\mathrm{E}}\left(\mathrm{Q}(\mathrm{u}, \mathrm{u}), \mathrm{r}^{*} \mathrm{R}\right)$.

Lemma 2.3. If $u_{n} \in B_{E}(0, R) \cap B_{E}(u, R), u \in B_{E}(0, R)$, and $v_{i} \frac{\partial u_{n}}{\partial x_{i}} \in B_{E}\left(v_{i} \frac{\partial u}{\partial x_{i}}, R^{* *}\right)$, with $\mathrm{r}^{* *}=\mathrm{r} * \mathrm{R}+\mathrm{R}+\mathrm{nR}^{* *} \geq 0$ such that

$$
0<\int_{\mathrm{B}_{3 \mathrm{R}}} \int_{|\mathrm{p}|=1}\|(v-z)\| \mathrm{dpd} z<\infty \quad \text { and } \quad 0<\int_{\mathrm{B}_{3 \mathrm{R}}} \int_{|\mathrm{p}|=1}\|(v-z)\| \mathrm{d} \mathrm{pd} v<\infty,
$$

then $\mathrm{J}\left(\mathrm{u}_{\mathrm{n}}\right) \in \mathrm{B}_{\mathrm{E}}\left(\mathrm{J}(\mathrm{u}), \mathrm{r}^{* *}\right)$ 


\section{Proof.}

$$
\begin{aligned}
& \left|J\left(u_{n}\right)-J(u)\right|=\left|u_{n}+v \cdot \nabla_{x} u_{n}-Q\left(u_{n}, u_{n}\right)-\mathfrak{u}-v \cdot \nabla_{x} u+Q(u, u)\right| \\
& \leq\left|u_{n}-u\right|+\left|Q\left(u_{n}, u_{n}\right)-Q(u, u)\right|+\left|v \cdot \nabla_{x} u_{n}-v \cdot \nabla_{x} u\right|, \quad \text { luego: } \\
& \left\|J\left(u_{n}\right)-J(u)\right\|_{L^{1}\left(\Omega \times B_{3 R}(0)\right)} \leq\left\|u_{n}-u\right\|_{L^{1}\left(\Omega \times B_{3 R}(0)\right)}+\left\|Q\left(u_{n}, u_{n}\right)-Q(u, u)\right\|_{L^{1}\left(\Omega \times B_{3 R}(0)\right)} \\
& +\left\|\sum_{i=1}^{n} v_{i} \frac{\partial}{\partial x_{i}}\left(u_{n}-u\right)\right\|_{L_{1}\left(\Omega \times B_{3 R}(0)\right)}
\end{aligned}
$$

now calculating $\frac{\partial J(\mathfrak{u})}{\partial x_{i}}$, we obtain that:

$$
\begin{aligned}
\left\|J\left(u_{n}\right)-J(u)\right\|_{E} & \leq\left\|u_{n}-u\right\|_{E}+\left\|Q\left(u_{n}, u_{n}\right)-Q(u, u)\right\|_{E}+\sum_{i=1}^{n}\left\|v_{i} \frac{\partial}{\partial x_{i}}\left(u_{n}-u\right)\right\|_{E} \\
& \leq r^{*} R+R+n R^{* *}=r^{* *}
\end{aligned}
$$

therefore $J\left(u_{n}\right) \in B_{E}\left(J(u), r^{* *}\right)$.

Lemma 2.4. The operator $\mathrm{J}: \overline{\mathrm{U}} \longrightarrow \mathrm{C}$ is compact. Dunford-Pettis Criterion will be applied, see [8], in fact:

i) $\int_{\Omega}|\mathrm{J}(\mathrm{u})| \mathrm{du} \leq \int_{\Omega} 2 \mathrm{R} \mathrm{du}=2 \mathrm{R} \mathrm{m}(\Omega) \leq 2 \mathrm{R} \delta$, defining $\varepsilon=2 \mathrm{R} \delta$, then the existence of $\delta$, such that if $\mathrm{m}(\Omega) \leq \delta$, then $\int_{\Omega}|\mathrm{J}(\mathrm{u})| \mathrm{d} u \leq \varepsilon$.

ii) Given $\varepsilon^{*}>0$, exists a closed, $\mathrm{F} \subset \Omega$ such that if $\mathrm{m}(\mathrm{F})<\infty$, then $\int_{\Omega-\mathrm{F}}|\mathrm{J}(\mathrm{u})| \mathrm{d} u \leq$ $2 R m(\Omega-F) \leq 2 R \varepsilon^{*}$, if we defined $2 R \varepsilon^{*} \leq \varepsilon$, then $\int_{\Omega-F}|J(u)| d u \leq \varepsilon$.

Lemma 2.5. For every $\mathfrak{u} \in \partial \mathrm{U}$ you have $\mathfrak{u}=\mathrm{J}(\mathfrak{u})$. In fact, if $\mathfrak{u} \in \partial \mathrm{u}$, then $\mathfrak{u}=e^{-|v|^{2}}$, and:

$$
\mathrm{J}(\mathfrak{u})=\mathfrak{u}+v \cdot \nabla_{x} u-Q(u, u)=u=e^{-|v|^{2}}
$$

then for every $u \in \partial u \quad y \quad \lambda \in(0,1)$ must be $\mathfrak{u} \neq \lambda \mathrm{J}(\mathfrak{u})$.

Therefore the nonlinear alternative Leray-Schauder type, see [1], page 48, we conclude that there is a fixed point of the operator J a solution resulting from (1.1). 
Received: October 2012. Accepted: September 2013.

\section{References}

[1] Agarwal R., Meeman M. and Oregan D., Fixed Point Theory and Applications, Cambridge University Press, (2004).

[2] Arkeryd L. and Nouri A., The Stationary Boltzmann equation in the Slab with given weighted masss for hard and Soft Forces, Annals Scuola Normal Superior. PISA Cl. Sci., vol 27,533-556, (1998).

[3] Arkeryd L. and Nouri A., On the Stationary Povzner equation in three Space Variables, Journal of Mathematics Kyoto University, vol 39,115-153,(1999).

[4] Arkeryd L. and Nouri A., L' Solutions to the Stationary Boltzmann equation in a Slab, Annals Faculty Science of Toulose math., vol 9, 375-413, (2000).

[5] Arkeryd L. and Nouri A., The Stationary Boltzmann Equation in $\mathbb{R}^{n}$ with given indata, Ann. Scuola Norm Sup. di Pisa.

[6] Arkeryd L. and Nouri A., The Stationary Boltzmann equation in $\mathbb{R}^{n}$ with given in data, Annals Scuola Normal Sup. Pisa, vol 31, 1-28, (2002).

[7] Arkeryd L. and Nouri A., The Stationary nonlinear Boltzmann equation in a Coutle setting; isolated solutions and non-uniqueness, Preprint, (2003).

[8] Brezis H., Functional Analysis, Sobolev Spaces and Partial Differential Equations, Springer Verlag, (2010).

[9] Costa David G., An Invitation to Variational Methods in Differential Eqyuations, Birkhauser, (2007).

[10] Grad H., High Frequency Sound Recording According to Boltzmann equation, SIAM J. Appl. Math., 14, 935-955, (1966).

[11] Maslova N., Non linear evolution equations Kinetic Approach, Series on Advances in Mathematics for Applied Sciences, vol. 10, World Scientific.

[12] Panferov V., On the existence of Stationary solutions to the Povzner equation in a Bounded Domain, Preprint, (2000).

[13] Sone Y., Kinetic Theory and Fluid Dynamics, Birkhauser, Boston, (2002).

[14] Ukay S., Yang T. and Zhao H., Stationary solutions to the exterior problems for the Boltzmann Equation Existence, Discrete and Continuous Dynamical Systems., vol. 23, No.152, January and February (2009). 